\title{
Observer-based Transient Frequency Drift Compensation in AC Microgrids
}

\author{
Ángel Navarro-Rodríguez, Student Member, IEEE, Pablo García, Member, IEEE, Ramy Georgious, Student \\ Member, IEEE, Jorge García, Senior Member, IEEE, and Sarah Saeed, Student Member, IEEE
}

\begin{abstract}
This paper proposes a novel solution for transient frequency compensation in weak 3-phase Microgrids (MGs) based on a Luenberger observer and a transient frequency detector. Unlike in conventional grids, the low inertia of the generators coupled to a MG could make their rotor speeds to be affected by load changes, varying the grid frequency and compromising the grid quality and stability. This problem has been approached in the literature by the Virtual Inertia (VI) concept. However, the existing solutions are affected by the decoupling of the grid frequency reference and the frequency estimation bandwidth. The proposed paper addresses these problems by the use of a transient frequency drift estimator based on a transient detector and a Luenberger type observer that provides a nearly-zero lag frequency estimation. The proposed alternative is analytically compared with the existing techniques and validated through simulation and exhaustive experimental results in an islanded MG. The developed method enables a $1 \mathrm{~Hz}$ reduction in the transient frequency deviation when compared with the existing alternatives and improves the system stability.
\end{abstract}

Index Terms-AC-DC, DC-DC power converters, Energy storage, Frequency control, Microgrids, Observers, Power control, Power quality, Power system dynamics.

\section{INTRODUCTION}

$\mathbf{T}$ HE weakness, quality and stability problems associated to Microgrids (MGs) have been considered since they emerged, demanding a significant research effort [1]-[3]. Distribution Static Synchronous Compensator (D-STATCOM) with Energy Storage, or simply Energy Storage System (ESS), are the preferred solution for power quality enhancement involving active power exchange in MGs [4]-[6].

Some examples of ESS use can be found for frequency profile enhancement [7], [8]. In these applications, the ESS works in coordination with a power plant, using a communication link and providing access to the system variables. Often, the ESS participates both in the power sharing during steady state and transients. In [3], an ESS system was proposed for frequency compensation limited to the operation during transients. However, the proposed solution relies on the commu-

The present work has been partially supported by the predoctoral grants program Severo Ochoa for the formation in research and university teaching of Principado de Asturias PCTI-FICYT under grants ID BP13-138, BP14135 and BP16-133. This work also was supported in part by the Research, Technological Development and Innovation Program Oriented to the Society Challenges of the Spanish Ministry of Economy and Competitiveness under grants ENE2013-44245-R and ENE2016-77919-R and by the European Union through ERFD Structural Funds (FEDER).

A. Navarro-Rodríguez, P. García, R. Georgious, J. García and S. Saeed are with the Department of Electrical, Computer and System Engineering, University of Oviedo, Gijón, 33204, Spain (e-mail: navarroangel@uniovi.es, garciafpablo@uniovi.es, georgiousramy@uniovi.es, garciajorge@uniovi.es, hazkialsarah.uo@uniovi.es). nication with the generation system. The communication-less frequency compensation in MGs has been already proposed, being one of the most common solutions the Virtual Inertia (VI) [9]-[16].

The absence of communications leads to three main constraints: 1) The grid frequency must be estimated from the voltage signals at the point of common coupling (PCC). Methods such as Phase-Locked Loop (PLL) [17], [18], or more robust techniques as Frequency-Locked Loop (FLL) [19], can be used. However, they are characterized by relatively slow dynamics which limits the compensator controller bandwidth. 2) Improved load-disturbance rejection capability needs for a derivate action to fasten the controller response, however this is prone to noise in the estimated frequency signal. 3) Avoiding power sharing during steady state might be compromised with varying grid frequency reference.

The present paper will focus on the design of an improved transient frequency drift compensator (TFDC) for its application in weak MGs. The TFDC will be implemented by means of a D-STATCOM with ESS, being the proposed method agnostic with respect to the ESS technology. Although other enhanced solutions appear in the literature [20]-[24], in the present study, two of the main existing limitations for frequency compensation are addressed: the decoupling of the grid frequency reference and the low bandwidth of grid frequency estimators. The performance achieved by existing feedback control has been improved by the development of a transient observer, formed by a novel transient detection method based on signal correlation that effectively decouples the grid reference frequency from the compensator inputs, and a Luenberger-based observer that provides a nearly-zero lag frequency estimation and allows to increase the phase margin in the frequency controller. Among the contributions in the present paper, it is worth to highlight: 1) an exhaustive system characterization and disturbance rejection analysis of the existing methods compared with the proposed TFDC, 2) a novel method for the detection of the transient frequency and 3) the implementation of a novel enhanced solution based on the use of a Luenberger type observer [25] for the grid frequency estimation that allows for a reduction of the compensator phase lag, mitigating the dependence on the derivative factor associated to VI.

The proposed techniques have been compared with the existing ones, analytically and experimentally, in an isolated MG with resistive and Constant Power Loads (CPL), evincing the limitations of VI and existing frequency estimators when used for transient frequency compensation. A normalized 
performance comparison has been derived from the analysis, establishing a benchmark for the selection of the most suitable method depending on the application. Under the conditions used in this paper, a reduction of $1 \mathrm{~Hz}$ in the initial frequency drift compared with the existing techniques is achieved. Thus, the contributions in this paper lead to the possibility of faster frequency compensation in weak grids even with a reduced FLL or PLL bandwidth.

The paper is organized as follows. Section II formulates the dynamic frequency drift problem. Section III covers the control structure of the TFDC. Section IV details the model and use of the Luenberger based observer. Section V shows the experimental results.

\section{Problem Definition and System Modeling}

Deviations on power grid nominal parameters can lead to non-optimal operation or malfunction of grid connected elements. Moreover, the violation of regulations could trigger the system protections, compromising the grid power quality and stability. As a reference, the default IEEE regulation for distributed resources is summarized in Table I, where $f_{e}$ and $f_{n}$ are the instantaneous grid frequency and the nominal grid frequency respectively.

TABLE I

DEFAULT CLEARING TIMES UNDER ABNORMAL FREQUENCY OPERATION BASED ON IEEE STD 1547A-2014 [26]

\begin{tabular}{c|c}
\hline Frequency [Hz] & Clearing Time [s] \\
\hline$f_{e}<f_{n}-3$ & 0.16 \\
$f_{e}<f_{n}-0.5$ & 2 \\
$f_{e}>f_{n}+0.5$ & 2 \\
$f_{e}>f_{n}+2$ & 0.16 \\
\hline
\end{tabular}

\section{A. Problem description}

MGs and distribution networks are often governed by low inertia synchronous generators, being the grid active power related to their rotating speed. In weak grids, the connection and disconnection of the different grid elements, as power generators and loads, may cause variations on the voltage magnitude and grid frequency. This problem is illustrated in Fig. 1, where the load power disturbance and the induced frequency drift is shown. This issue can be mitigated by using a solution capable of injecting power to the grid with a much faster dynamic response than the grid generators, thus compensating the power mismatch.

\section{$B$. Integration of the proposed solution in the $M G$}

Fig. 1 shows the proposed solution integrated in an experimental isolated weak MG. The MG consists in a single node isolated 3-phase MG, governed by a distributed synchronous generator, feeding both passive and active loads. Such a generator operates in slack mode when the MG is disconnected from the utility grid, and presents a high output impedance $\left(R_{g}, L_{g}\right)$ and low mechanical inertia. The synchronous generator is implemented by a permanent magnet synchronous generator (PMSG) mechanically coupled to a

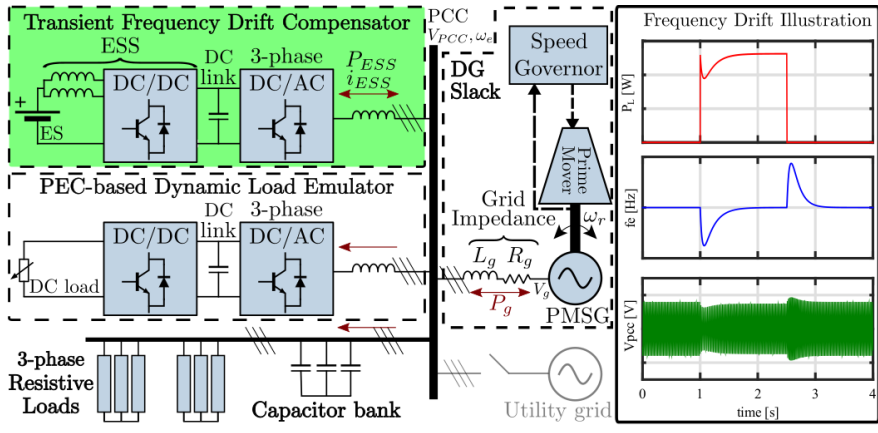

Fig. 1. Integration of the system under study in an isolated MG governed by a distributed synchronous generator operating in slack mode. The frequency drift contingency is illustrated on the right.

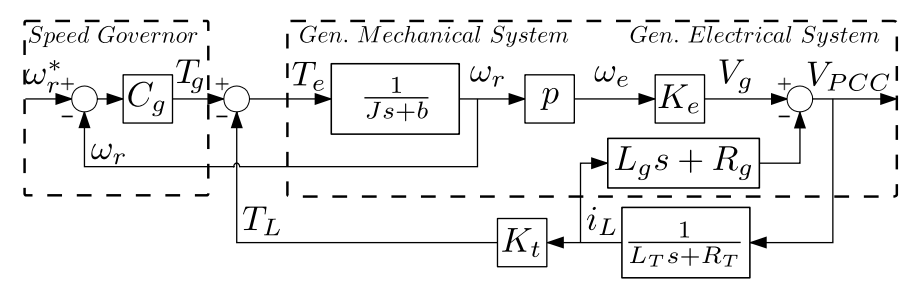

Fig. 2. Equivalent block diagram of a PM synchronous generator supplying constant-impedance type loads.

permanent magnet synchronous motor (PMSM), acting as a speed governor. In order to make the setup to be as close as possible to a generic MG, both passive load, consisting of 3phase resistors, and tightly regulated dynamic loads operated in Constant Power Load (CPL) mode, are present in the MG. CPL are emulated by by a grid tied AC/DC IGBT inverter coupled to a DC/DC converter that feeds a DC load.

The proposed compensating solution, highlighted in green, is integrated into a D-STATCOM, consisting of a 3-phase IGBT inverter coupled through a DC link to an ESS, and a bidirectional DC/DC boost IGBT converter in 2 branch interleaved configuration. The proposed topology is able to both inject or absorb active/reactive power, thus being able to assist the distributed generator during transients due to load disturbances, smoothing the frequency dynamic response. During steady state operation, the TFDC algorithm should be idle, being the stationary control out of the scope of this paper.

\section{Generator model}

For high-performance frequency compensation, the system dynamics must be deeply analyzed. Moreover, the system dynamic modeling becomes mandatory when using observerbased techniques. Fig. 2 shows the equivalent system of a synchronous generator coupled to a speed governor. $\omega_{r}$ is the rotor mechanical speed, $C_{g}$ the governor controller, $T_{g}$ the governor injected torque, $J$ and $b$ the generator inertia and friction coefficients, $K_{e}$ and $K_{t}$ the generator back EMF and torque constant and $p$ the number of pole pairs. The electrical load is represented by the parameters $R_{T}$ and $L_{T}, i_{L}$ is the load current and $T_{L}$ is the equivalent load torque. $R_{g}$ and $L_{g}$ represent the grid impedance, in this case, the generator stator impedance. 
The generator mechanical system is coupled to its electrical counterpart, thus any change or disturbance in the electrical grid will be reflected in its mechanical system. When an electrical load demanding active power is suddenly connected to the grid, it will generate an equivalent load torque proportional to the load current at the generator's shaft. This torque will be seen as a disturbance by the governor, causing a speed variation which depends on the mechanical inertia and the governor control system. Considering the control system of the governor as a PI regulator in the ideal form $C_{g}(s)=K p \cdot\left(1+\frac{1}{s T i}\right)$, and neglecting the dynamic contribution of the generator electrical subsystem, the disturbance transfer function $D_{g}$ is given by (1).

$D_{g}(s)=\frac{-\omega_{r}(s)}{T_{L}(s)}=\frac{G_{g e n}(s)}{1+C_{g}(s) G_{g e n}(s)}=\frac{\frac{1}{J} s}{s^{2}+\frac{K_{p}+b}{J} s+\frac{K_{p}}{T_{i} J}}$

For future use and analysis in this paper, $D_{g}(s)$ is reformulated by a general $2^{\text {nd }}$ order expression (2).

$$
D_{g}(s)=\frac{k_{g} \omega_{n g}^{2} s}{s^{2}+2 \xi_{g} \omega_{n g} s+\omega_{n g}^{2}}
$$

If the governor is considered as an ideal PI regulator, the parameters are equivalent to $k_{g}=\frac{T_{i}}{K_{p}}, \omega_{n g}=\sqrt{\frac{K_{p}}{T_{i} J}}$ and $\xi_{n g}=\frac{b+K_{p}}{2 J \omega_{n g}}$.

It is worth noting that not only the frequency but also the generator voltage is proportional to the mechanical speed through the back electromotive force constant. Therefore, the compensation could positively affect both the active and reactive power sharing.

\section{Transient Frequency Drift Compensation}

The basic idea of the dynamic frequency drift compensation consists in maintaining the active power balance between the grid elements during transients. In the case under study, the transient active power mismatch between the generation and consumption in the grid will depend on the grid equivalent inertia determined by the synchronous generators. For that reason, one of the first ideas that emerged consisted in introducing a VI by using an ESS that emulates the behavior of a synchronous generator, known in the literature as Virtual Synchronous Machine (VSM) [20]. The controller is defined by the pseudo-derivative feedback regulator (PDF), [27], given by (3).

$$
T_{E S S}(t)=k_{p E S S}\left(w_{e}^{*}(t)-w_{e}(t)\right)-J_{E S S} \cdot \frac{d}{d t} w_{e}(t)
$$

Where $T_{E S S}$ is the equivalent torque, $k_{p E S S}$ the damping coefficient, $J_{E S S}$ the virtual inertia gain, $w_{e}$ the instantaneous grid frequency and $w_{e}^{*}$ the grid frequency reference. The conditions and constraints of the proposed communication-less solution are the following:

- There is not communication between the proposed solution and any other system at the grid.

- Only the currents and voltages at the PCC are available.

- The control relies on the the estimated grid frequency.

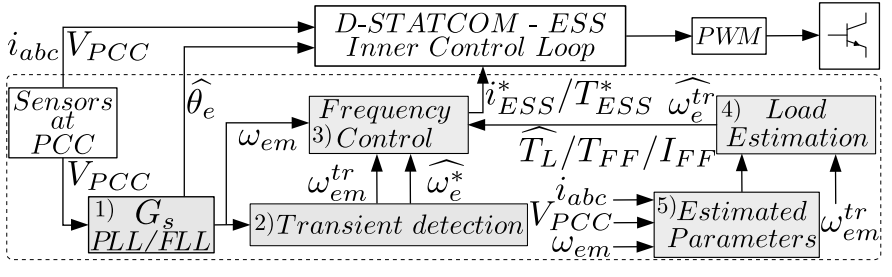

Fig. 3. Simplified control scheme for the proposed system.

- The paper is focused on improving the transient frequency drift, limiting the power exchange between the proposed system and the grid.

- The grid frequency command in the MG $\left(w_{e}^{*}\right)$ is unknown.

- The possibility of binding the proposed solution to a significant load in the grid, gaining access to the load current, is also considered.

Under these constraints, the main problems to be tackled are summarized as:

- The frequency has to be estimated from electrical variables. The effects of measurement noise and delays as well as grid distortion over the used frequency estimation techniques must be considered.

- The transient state has to be detected in order to avoid active power injection during the steady-state. The steadystate frequency command must be decoupled.

- The use of derivative terms may compromise the reliability under noisy conditions.

\section{A. Proposed Control System}

The proposed control system scheme is shown in Fig. 3. Besides the internal current control loops, five blocks can be identified forming the compensator control.

1) Grid synchronization and frequency estimation: It provides the estimated grid angle $\left(\widehat{\theta}_{e}\right)$ and the estimated grid frequency $\left(\omega_{e m}\right)$. It is based on PLL, DSOGI-FLL techniques [17]-[19] or any other frequency estimation method. The transient performance has been experimentally evaluated in Fig. 4, using the generator speed measurement given by a resolver as a reference for red the comparison. Both PLL with low pass filter and FLL are good candidates for the frequency estimation. Due to its robustness under distorted grids, a DSOGI-FLL will be used in this paper. Its dynamic transfer function will be referred henceforth as $G_{s}(s)$, modeled as the $2^{\text {nd }}$ order system approximation in (4), obtained by curve-fitting methods.

$$
G_{s(s)}=\frac{\omega_{e m}}{\omega_{e}}=\frac{k_{s} \omega_{n s}^{2}}{s^{2}+2 \xi_{s} \omega_{n s} s+\omega_{n s}^{2}}
$$

Where $k_{s}$ is the sensor gain, 1 by default, and $\omega_{n s}, \xi_{s}$ are the natural frequency and damping factor of the system respectively.

2) Transient detection: To avoid steady-state compensation, the detection of the frequency transients is required and the actual grid frequency command has to be decoupled. The proposed method is detailed at section III-B. 


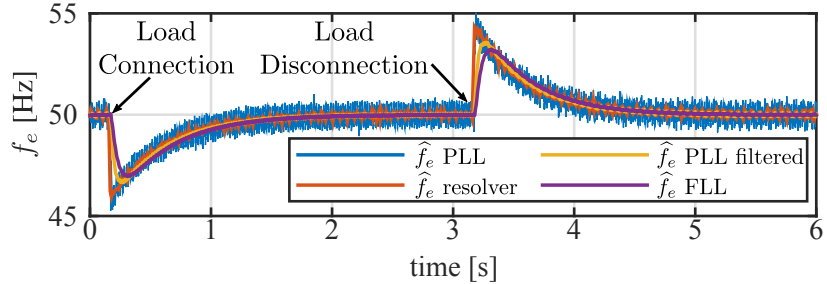

Fig. 4. Experimental results for the frequency drift measurement at the PCC under the connection and disconnection of a resistive load without compensation. The used setup is defined by Fig. 1 and Table II.

3) Frequency control: The main block of the control system consist in a feedback regulator able to provide a current or torque reference for the D-STATCOM $\left(i_{E S S}^{*}, T_{E S S}^{*}\right)$, using the error between the frequency reference and the frequency estimation. Details for the feedback based control are given at section III-C.

4) Load disturbance estimation: When grid parameters are known or estimated, it is possible to generate a load estimation and use it as a feed-forward, either in terms of power, torque or current $\left(T_{F F}, I_{F F}\right)$ to improve the dynamic response [8]. In this study, two options have been considered. One is based on the current measurement, developed at section III-D, and another relying on a Luenberger-based observer, considered at section IV.

5) Estimated parameters: The necessary information for the implementation of the load disturbance observer should be estimated either on-line or off-line. In the scope of this paper all the parameters will be known or obtained off-line.

\section{B. Transient detection}

The transient detection issue has been already covered in previous literature by 1) using a transient detection window [3], and 2) an open loop estimator to extract the frequency reference considering a droop-controlled grid [28]. In the first case, a simple comparison of the feedback frequency and a threshold determines a transient window. However, delays, noise and harmonic distortion in the frequency estimator may lead to incorrect performance. In the second case, the open loop estimator leads to errors with any change in the estimator parameters. In this paper, a method based on the correlation of the measured frequency with a signal of period $T$ and zero average is proposed (5).

$C \omega_{e}(t)=\int_{0}^{T} x(t) f(t) d t \rightarrow C^{2} \omega_{e}(t)=\left(\int_{0}^{T} x(t) f(t) d t\right)^{2}$

where $C \omega_{e}$ is the correlation result, $x(t)$ is $\omega_{e m}$ and $f(t)$ could be any function having zero average. For the sake of simplicity, a sine type function is used.

Assuming that the grid frequency reference will have a slow variation compared to the integration interval, $T$, the correlation function will give low values during the steady state. On the other hand, when a transient frequency drift occurs, it will lead to larger values. The resulting behavior is similar to the use of a derivative, but without the associated

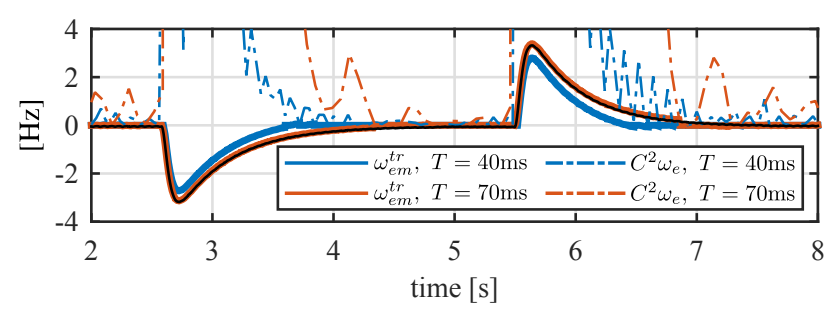

Fig. 5. Experimental results for the transient frequency estimation using the proposed method. Evaluation under connection and disconnection of a resistive load at the PCC without compensation. The setup is defined by Fig. 1 and Table II.

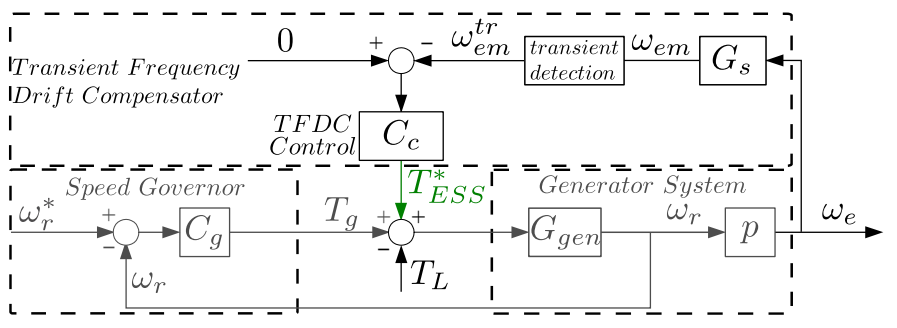

Fig. 6. Proposed controller for transient frequency drift compensation in the grid model block diagram. The speed governor and generator blocks match the system presented in Fig. 2. The torque provided by the TFDC is highlighted in green.

noise problems. Using the correlation squared value, (5), a transient window is generated and any frequency out of that window is considered to be the the grid frequency reference $\left(\omega_{e}^{*}\right)$. The last frequency value before the transient detection is considered as the estimated reference frequency $\left(\widehat{\omega_{e}^{*}}\right)$. The transient detector output is the transient frequency $\left(\omega_{e m}^{t r}\right)$, obtained as $\omega_{e m}^{t r}=\omega_{e m}-\widehat{\omega_{e}^{*}}$.

The obtained experimental results for the estimated transient frequency are shown at Fig. 5. Two different $T$ values are used for the estimation: $T=40 \mathrm{~ms}$ and $T=70 \mathrm{~ms}$. The correlation value is scaled by 10 to simplify the representation. Threshold value is set to 5 and the FLL bandwidth is set to $5 \mathrm{~Hz}$. The results are compared with respect to the measured frequency, from which the reference value has been subtracted.

\section{Frequency feedback based control}

The feedback based frequency control is shown in Fig. 6. The TFDC controller $C_{c}$, uses the estimated transient frequency $\left(\omega_{e m}^{t r}\right)$, obtained from the frequency sensor and the transient detection block, to provide a control action by means of an equivalent torque command $\left(T_{E S S}^{*}\right)$.

Once the TFDC is included in the system, the disturbance rejection transfer function $\frac{\omega_{r}}{T_{L}}$ is given by (6)

$$
D_{c}(s)=\frac{\omega_{r}(s)}{T_{L}(s)}=-\frac{D_{g}(s)}{1+D_{g}(s) \cdot C_{c}(s) \cdot G_{s}^{\prime}(s)}
$$

where $G_{s}^{\prime}(s)=G_{s}(s) \cdot p, p$ is the number of pole pairs of the machine and $G_{s}(s)$ the sensor transfer function. $D_{g}(s)$ is the generator disturbance transfer function in (2). The obtained expression only applies during the transient, assuming the grid frequency reference has been already decoupled. 


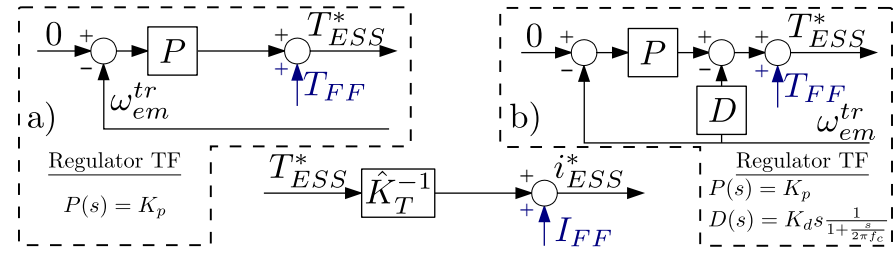

Fig. 7. Control structure for frequency drift compensation. a) Proportional (P) regulator; b) Pseudo-derivative feedback (PDF) structure. An optional load feed-forward can be applied either in terms of torque, $T_{F F}$, or current, $I_{F F}$.

Fig. 7 shows the general scheme for the frequency controller $C_{c}$, including the torque to current conversion. A feed-forward to improve the control is considered as an optional functionality $\left(T_{F F}, I_{F F}\right)$.

It is worth noting that PI regulators are not considered in this study as the use of an integral action in the controller, even if it can improve the steady state disturbance rejection drastically, would also lead to the unavoidable share of power during steady state. P and PDF options will be analyzed. The two alternative transfer functions of the feedback frequency controller $C_{c}(s)$ are defined by (7), P, and (8), PDF regulator, assuming the input as $-\omega_{e m}^{t r}$.

$$
C_{c}(s)=K_{p} \quad \text { (7) } \quad C_{c}(s)=K_{p}+K_{d} s \frac{2 \pi f_{c}}{2 \pi f_{c}+s}
$$

Where $K_{p}$ and $K_{d}$ are the proportional and derivative gains of the compensator. These two gains are equivalent to the damping coefficient $k_{p E S S}$ and the virtual inertia gain $J_{E S S}$ in (3) respectively. The variable $f_{c}$ is the cut-off frequency of the low-pass filter associated to the derivative term.

The different alternatives are compared based on their dynamic stiffness, defined as (9).

$$
S_{c}(s)=\frac{T_{L}(s)}{\omega_{r}(s)}=\frac{1+D_{g}(s) \cdot C_{c}(s) \cdot G_{s}^{\prime}(s)}{D_{g}(s)}
$$

Fig. 8 shows the dynamic stiffness evaluated in frequency domain and the corresponding transient response in time domain for two different $\mathrm{P}$ and PDF settings. Two different proportional gains providing two different gain margins (GM) are used. In the case of the PDF, a minimum phase margin of $60^{\circ}$ is set at the open-loop crossover frequency in both cases. The graph shows the benefits of an increased proportional gain and the improved disturbance rejection capability provided by the inclusion of the differential term. As shown in time domain, the predicted benefits for including the differential term are translated to a reduced initial overshoot when compared to the $\mathrm{P}$ method.

Finally, the experimental results for the feedback-based compensation are shown in Fig. 9. A good agreement with respect to the previous theoretical discussion can be observed.

\section{Load feed-forward and state observation}

Enhanced dynamic response for the transient frequency drift controller can be obtained by the use of feed-forward disturbance decoupling and the increase of the frequency estimation bandwidth by using a Luenberger type observer.

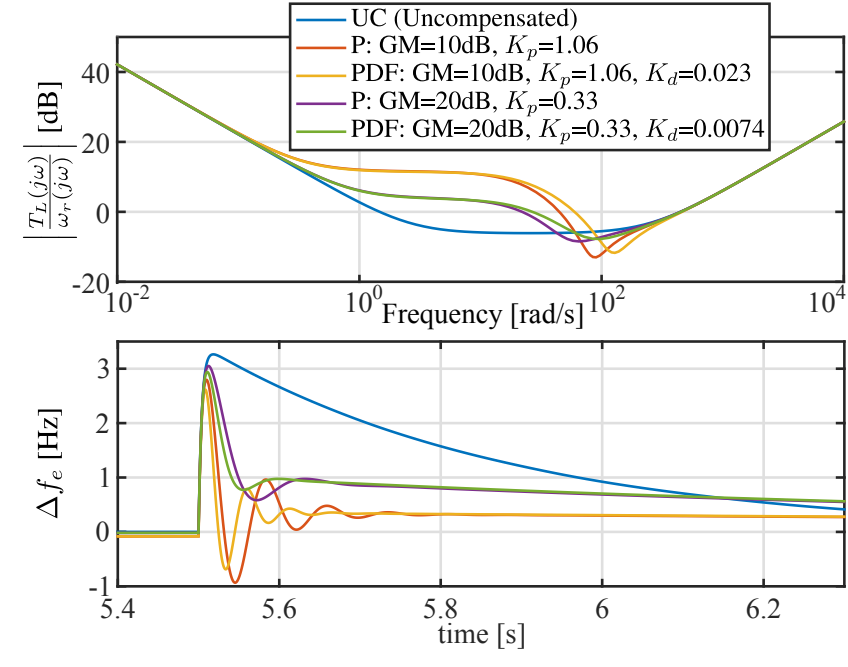

Fig. 8. Performance comparison of the P and PDF methods. Top: dynamic stiffness. Bottom: transient response.

If the load demand is available, it can be used as a feedforward for the controller, as shown in Fig. 7. Ideally, if the load is fully known, the frequency drift will be eliminated (grey line in Fig. 9). However, that solution would lead to the compensation of the whole load power also during steady state. In order to compensate only the transient and keep the grid frequency unalterable, the ESS has to provide the difference between the load power and the power drawn by the generator, i.e. the equivalent torque of the compensation system should be $T_{E S S}=T_{L}-T_{g}$. Nevertheless, in a communication-less system, the generator information is not available. However, the load information is still a valuable information as it is a derivative state of the frequency, allowing to anticipate the control reaction.

Fig. 9 shows the experimental performance comparison for the different feedback methods, considering different configurations, and the response when a load feed-forward is used. The shown signals correspond to the DSOGI-FLL frequency signal using a FLL $\mathrm{BW}=25 \mathrm{~Hz}$. UC stands for the uncompensated case. $\mathrm{P}+\mathrm{FF}$ and $\mathrm{PDF}+\mathrm{FF}$ are the combination of $\mathrm{P}$ and PDF with a load feed-forward respectively. The feed-forward is filtered by a $2^{\text {nd }}$ order high-pass filter with bandwidth $=0.5 \mathrm{~Hz}$. FC stands for the full load compensation obtained by equaling the D-STATCOM current reference to the load current. In this example, the load information comes from a load current sensor, which is a valid assumption if the proposed solution is to be coupled to a significant load.

\section{TRAnsient Frequency-Drift ObSERVER}

The main purpose of the observer is to provide a transient frequency-drift estimation by providing a nearly zero phase-lag $\widehat{\omega_{e}^{t r}}$ estimation within the observer bandwidth. This will boost the response of the TFDC, being able to compensate frequency drift transients usually affected by the PLL/FLL bandwidth restrictions. Additionally, the proposed observer also provides an estimation of the load disturbance that could be used for the feed-forward compensation replacing the $T_{F F}$ measurement 

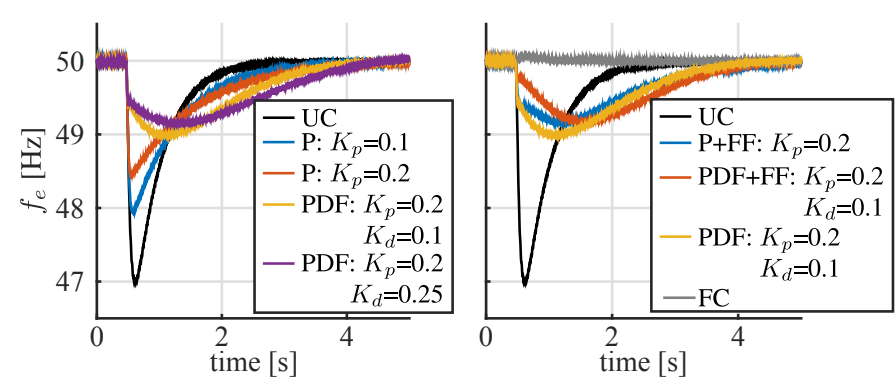

Fig. 9. Transient frequency drift compensation using different control methods under load connection transient. The setup is defined by Fig. 1 and Table II. Further details on the experimental setup are given at section V.

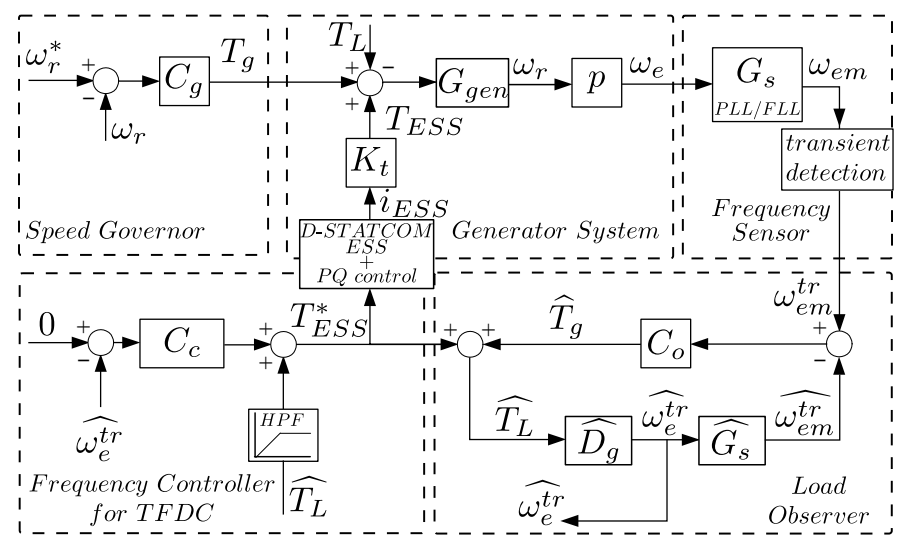

Fig. 10. Proposed observer control structure. The use of $\widehat{T_{L}}$ is optional.

by the estimated load $\widehat{T_{L}}$. Although the paper proposes the use of such a load estimation, $\widehat{T_{L}}$, it is not used or validated in this study, being part of future development. The proposed Luenberger-based observer control is shown in Fig. 10, where $C_{o}$ represents the transfer function of the observer regulator. It is worth to point out that in the case the observer is not used, $\omega_{e m}^{t r}$ will be the feedback variable for the close loop frequency controller $C_{c}$.

As the generator and governor parameters are unknown, the $C_{g}$ and $G_{g e n}$ transfer functions cannot be explicitly used in the proposed solution. However, the approximations proposed before for the sensor and the generator disturbance transfer functions (2), are appealing candidates for the observer implementation. The experimental comparison between the proposed approximations and the real systems are shown in Fig. 11. A really good matching is clearly observed. The observer is implemented using the state-space formulation corresponding to the model shown in Fig. 12. The model is defined by the state vector $x=\left[x_{1}, x_{2}, x_{3}, x_{4}\right]^{T}$, the input vector $u=\left[T_{L}\right]$ and the output vector $y=\left[\omega_{e}, \omega_{e m}\right]^{T}=\left[p \cdot x_{1}, x_{3}\right]^{T}$. The state, input, output and feed-forward matrices are defined in (10).

$$
\begin{gathered}
A=\left[\begin{array}{cccc}
-2 \xi_{g} \omega_{n g} & -\omega_{n g}^{2} & 0 & 0 \\
1 & 0 & 0 & 0 \\
0 & 0 & 0 & 1 \\
k_{s} p \omega_{n s}^{2} & 0 & -\omega_{n s}^{2} & -2 \xi_{s} \omega_{n s}
\end{array}\right], B=\left[\begin{array}{c}
k_{g} \omega_{n g}^{2} \\
0 \\
0 \\
0
\end{array}\right] \\
C=\left[\begin{array}{llll}
p & 0 & 0 & 0 \\
0 & 0 & 1 & 0
\end{array}\right]
\end{gathered}
$$
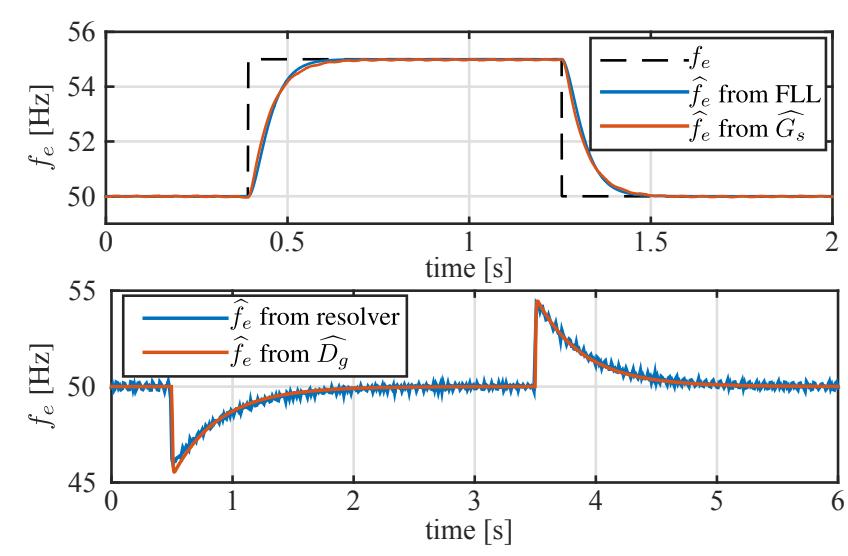

Fig. 11. Experimental results. Top: DSOGI-FLL response and its approximation using the $2^{\text {nd }}$ order transfer function $\widehat{G_{s}}$. Bottom: Open-loop response obtained with the estimation of the disturbance transfer function $\widehat{D_{g}}$ compared with the frequency measured by the generator resolver.

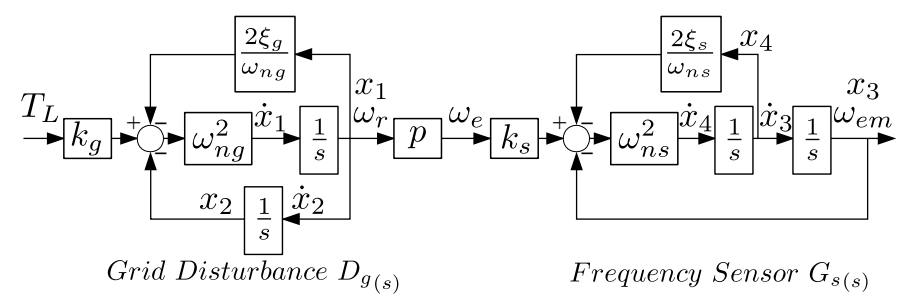

Fig. 12. Observer block diagram in state space form.

The dynamic stiffness for the proposed observer block diagram, assuming parameters matching between the real and the observed systems, is defined by (11).

$$
\frac{T_{L}(s)}{\omega_{e}(s)}=\frac{1+\widehat{D_{g}}(s) \cdot C_{o} \cdot \widehat{G_{s}}(s)}{1+\widehat{D_{g}}(s)\left(C_{c}(s)+C_{o}(s) \cdot \widehat{G_{s}}(s)\right)}
$$

The observer controller, $C_{o}(s)$, consists in a $4^{\text {th }}$ order transfer function, presenting the same order as the observed plant. It has been tuned in frequency domain using loop-shaping, trying to maximize the bandwidth while giving enough stability margin. The selected values are listed in Table II. The resulting frequency and transient responses comparing the proposed observer technique and the FLL feedback technique are shown in Fig. 13 and Fig. 14. The uncompensated and ideal sensor cases are shown as a reference. Ideal sensor case is defined as frequency feedback control with $G s(s)=1$, i.e., there is not phase lag introduced by the frequency estimation. As it can be seen, the use of the observer noticeably improves the initial transient response due to the compensated sensor lag. Moreover, in Fig. 14, the transient response of the design system (left $\omega n s=31.4 \mathrm{rad} / \mathrm{s}$ ), is compared with the response when the bandwidth of frequency sensor is reduced by four (right $\omega n s=7.85 \mathrm{rad} / \mathrm{s}$ ), without changing the observer regulator parameters. The proposed observer-based method still presents a better response than the alternatives.

\section{EXPERIMENTAL RESULTS}

The proposed frequency-drift compensation have been experimentally evaluated. The experimental grid has been presented in Section II, Fig. 1, and the relevant parameters 


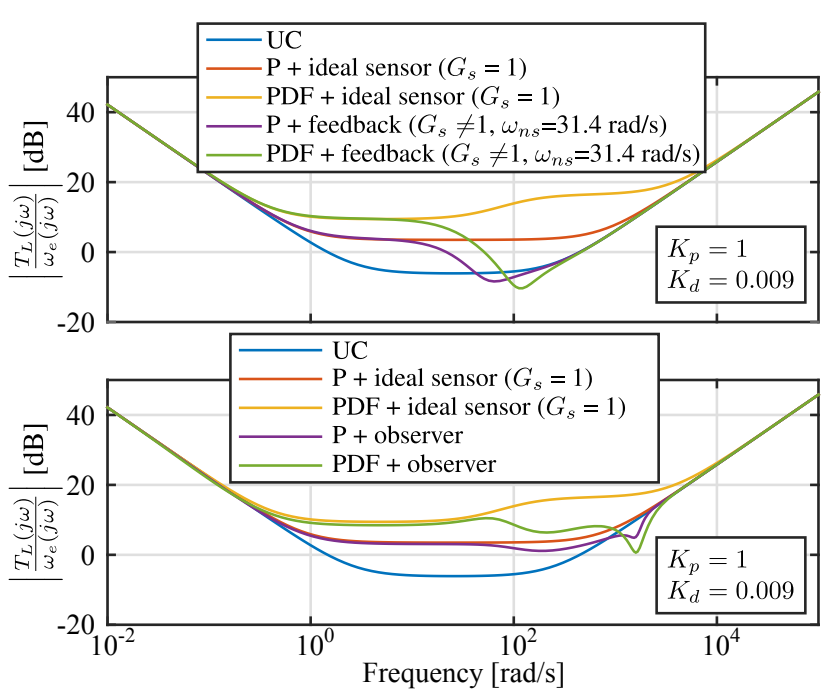

Fig. 13. Dynamic stiffness. Top: results for the feedback-based control. Bottom: observer-based control. The uncompensated and ideal sensor cases are shown as a reference.

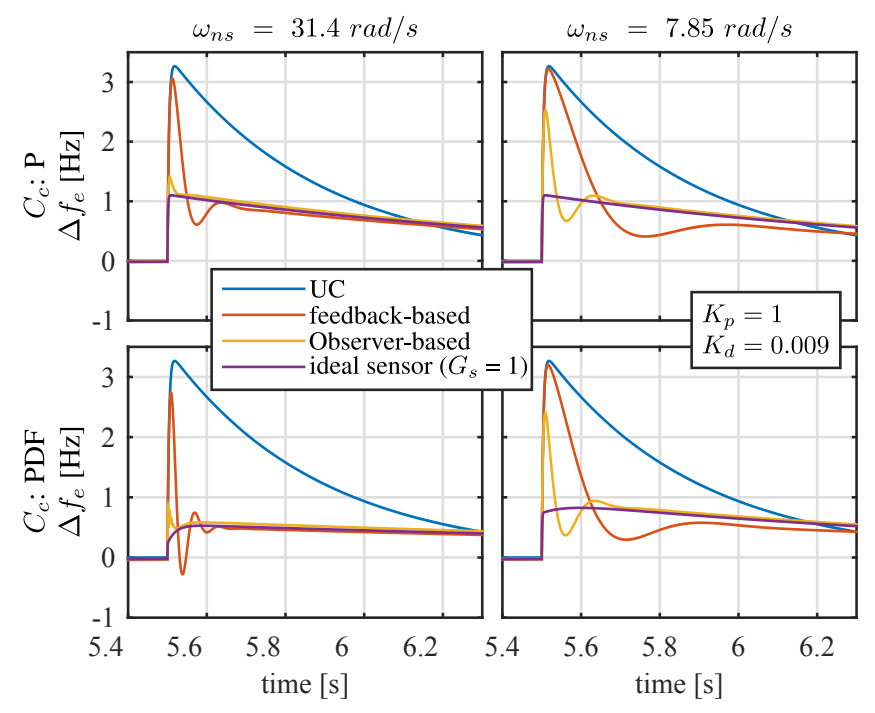

Fig. 14. Transient response comparison for different DSOGI-FLL bandwidth. Top: P controller. Bottom: PDF controller. Both cases are analyzed considering different feedback signals.

are given in Table II, including the $\mathrm{P}$ and PDF as well as the observer parameters used in the experimental tests. The equipment used to implement the experimental setup is shown in Fig. 15. The control system of the TFDC has been implemented in a TMS320F28335 DSC from Texas Instruments. The MG generator is emulated using 2 coupled PMSMs one acting as generator and other as the governor motor controlled by a commercial drive. The disturbances created by the different load types in the experimental MG, allow to test the effectiveness of the proposed observerbased compensator under a variety of conditions, enabling the comparison between the different existing methods which have been analyzed in this paper. It is worth noting that the grid frequency shown in the experimental results have been obtained using the speed resolver included in the industrial drive. The experimental results are obtained under two scenarios:

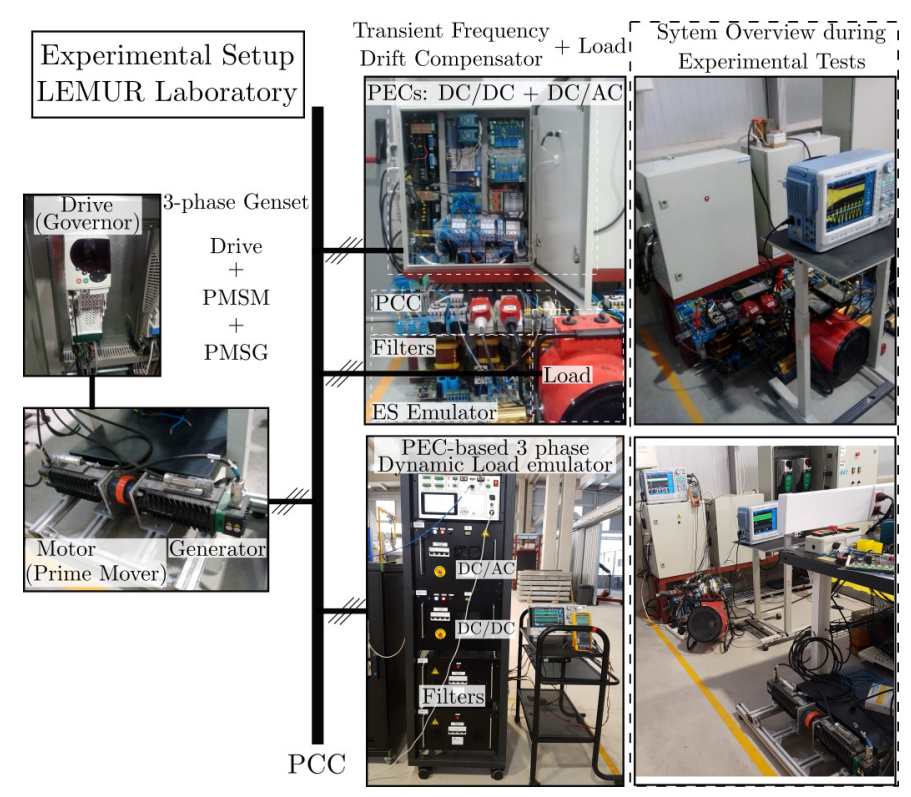

Fig. 15. Experimental prototype. Left: generator and drive used as the governor and the prime mover. Top center: TFDC and resistive load. Bottom center: dynamic load emulator based on PECs. Right: the setup during operation.

1) In the first scenario, the frequency variation for the evaluation of the TFDC is created by a load disturbance consisting in the connection and disconnection of a three-phase balanced resistive load. This test allows to characterized and compare the different methods evaluating the response under a step disturbance, avoiding to have unwanted disturbances in the middle of a transient.

2) In the second scenario, a more realistic load behavior is pursued. In this case, the load disturbance is a CPL generated by the PECs-based dynamic load emulator. This second scenario allows to demonstrate the advantages of the proposed observer and its viability in a realistic MG environment, where the PECs operation might be compromised by the transient frequency and voltage magnitude drifts.

A comparison of the transient response for the feedback and feed-forward methods is shown in Fig. 16 for the first scenario. The load feed-forward is implemented using the measured load current, applying a $1^{\text {st }}$ order high-pass filter with a cutoff frequency of $0.5 \mathrm{~Hz}$. The performance of the proposed observer-based solutions for the first scenario is shown in Fig. 17 compared with the feedback methods. As expected, feed-forward and the proposed observer-based methods have the best performance in terms of frequency compensation. Assuming that feed-forward methods need for extra sensors or/and communication, the proposed observer methods, that just depend on the voltage measurements at the PCC, are a promising alternative, offering an extra compensation at the beginning of the transient, reducing the maximum frequency deviation. In this particular case, for the selected parameters, the proposed methods $\mathrm{P}+\mathrm{Obs}$ and $\mathrm{PDF}+\mathrm{Obs}$ reduce the maximum frequency deviation in around $1 \mathrm{~Hz}$ when compared with P and PDF methods without observer, i.e., using $\omega_{e m}^{t r}$ instead 

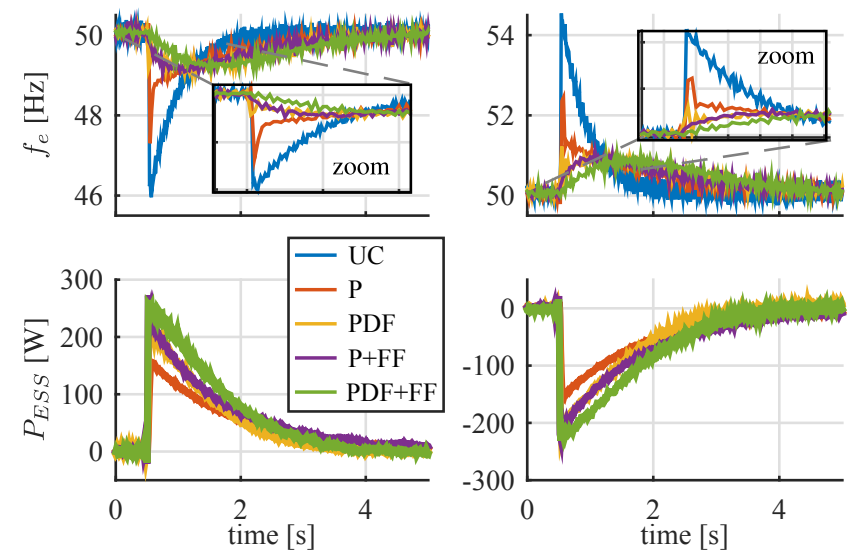

Fig. 16. Experimental results for the first scenario. Top: grid frequency obtained with the resolver. Bottom: power injected by the compensator. Left: load connection. Right: load disconnection.

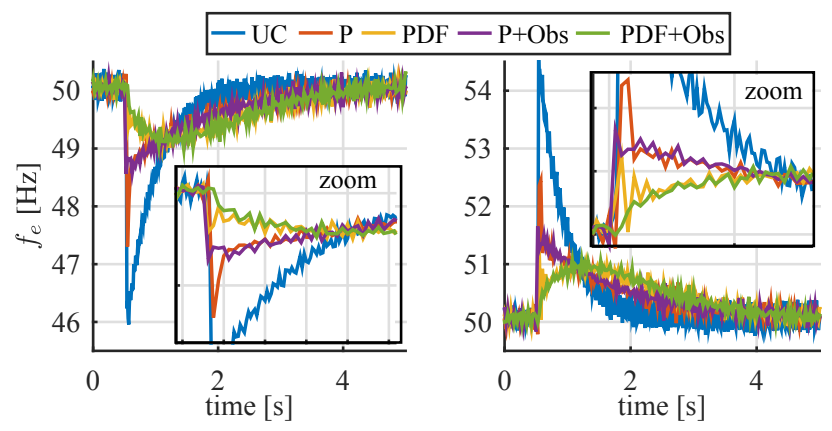

Fig. 17. Experimental results for the first scenario. Results using the proposed observer-based method. Grid frequency obtained with the resolver. Obs stands for observer-based compensation.

of $\widehat{\omega_{e}^{t r}}$.

Although it has not been applied or tested in this paper, the observer provides an additional useful information. The observed load current, obtained from the estimation of $\widehat{T_{L}}$, is represented in Fig. 18. As shown, the transient load disturbance can be estimated by a high-pass filter. This load estimation enables the implementation of a load feed-forward mechanism without the need for measuring the load current.

The results obtained for the second scenario using the PELsbased dynamic load emulator are shown in Fig. 19, for $\mathrm{P}$ and P+Obs methods, and in Fig. 21, for PDF and PDF+Obs. The frequency, the power drawn by the load emulator and the

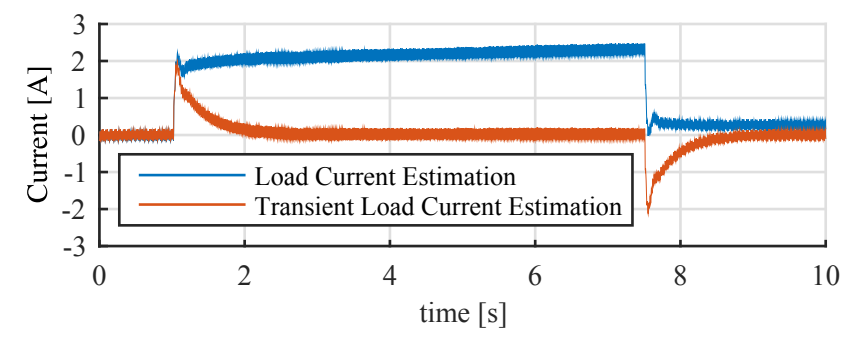

Fig. 18. Experimental results. Load current estimated by the observer. Full load current and transient load disturbance obtained by a $1^{\text {st }}$ order high-pass filter with $\mathrm{BW}=0.5 \mathrm{~Hz}$. The load current is $2.2 \mathrm{~A}$.
TABLE II

SYSTEM PARAMETERS

\begin{tabular}{c|c}
\hline MG Voltage ph-ph / $f_{n}$ & $98 \mathrm{~V}_{\mathrm{rms}} / 50 \mathrm{~Hz}$ \\
\hline 3-phase load & $32 \Omega$ \\
\hline BEMF / Torque constant / Poles & $98 \mathrm{~V} / \mathrm{krmp} / 1.6 \mathrm{Nm} / 3$ pairs \\
Mechanical parameters & $\mathrm{J}=0.0019 \mathrm{~kg} \cdot \mathrm{m}^{2}, \mathrm{~b}=0.2 \mathrm{Nms}$ \\
Electrical parameters & $L_{g}=6 \mathrm{mH}, R_{g}=2.1 \Omega$ \\
\hline Power / ESS Voltage & $20 \mathrm{~kW} / 140 \mathrm{~V}$ \\
DC link voltage / Capacitor & $300 \mathrm{~V} / 2 \mathrm{mF}$ \\
Grid connected AC filter & $R=0.2 \Omega, L=7.18 \mathrm{mH}$ \\
DC/DC converter filter & $R=0.1 \Omega, L=7.18 \mathrm{mH}$ \\
\hline TFDC DC/DC Current control & $\mathrm{BW}=500 \mathrm{~Hz}, K_{p i}=22, K_{i i}=13$ \\
TFDC DC link Voltage control & $\mathrm{BW}=50 \mathrm{~Hz}, K_{p v}=1, K_{i v}=175$ \\
\hline TFDC AC/DC Current control & $\mathrm{BW}=400 \mathrm{~Hz}, K_{p i}=22, K_{i i}=50$ \\
\hline TFDC Control Gains & $P: K_{p}=0.2$ \\
& $P D F: K_{p}=0.2, K_{d}=0.1$ \\
\hline Observer parameters & $k_{g}=0.78, \omega_{n g}=25.6, \xi_{g}=4.96$ \\
& $k_{s}=1, \omega_{n s}=31.4, \xi_{s}=1$ \\
\hline Observer $C_{o}$ coefficients & $K_{o}=10$, zeros $=[10,10,5,3.4] \cdot 10^{-3}$ \\
& poles $=[20,10,10,6.67] \cdot 10^{-4}$ \\
\hline Dynamic Load Emulator & Current BW $=500 \mathrm{~Hz}$ \\
& Power BW $=50 \mathrm{~Hz}$ \\
& Current limit $=10 \mathrm{~A}$ \\
& Nominal Power $=1 \mathrm{~kW}$ \\
\hline
\end{tabular}

power injected by the TFDC are represented. It is clearly seen the improvement in the the frequency profile with all methods when compared with the uncompensated case. In order to better compared them, Fig. 20 and 22 show the detailed view of the critical transients in the system.

The conclusions drawn from the experimental results can be summarized as: 1) for all the cases, the observer-based methods provide a reduction on the maximum frequency deviation when compared with the FLL feedback methods; 2) The maximum deviation is reduced by more than $1 \mathrm{~Hz}$ in the case of $\mathrm{P}+\mathrm{Obs}$ when compared with $\mathrm{P}$ and 3 ) when differential action is added, the proposed PDF+Obs method improves the frequency drift by more than $0.5 \mathrm{~Hz}$ when compared with the PDF. All this conclusions demonstrate the viability and superiority of the proposed methods for fast frequency drift compensation.

Finally, a last test was performed in order to evaluate the limits of the different compensation methods under significant load steps. Fig. 23 shows the response of the system under 3 load steps, for five cases: uncompensated, P, P+Obs, PDF and PDF+Obs. The figure does not only show the effect of load disturbances in the frequency but also in the voltage magnitude, which is affected due to the generator impedance and the coupling between its speed and voltage. As shown in Fig. 23, at $t=0.2 \mathrm{~s}$, a load step of $600 \mathrm{~W}$ is applied. Under this load condition, the load emulator trips for the uncompensated scenario due to an overcurrent as a result of the voltage magnitude and frequency distortion. At $t=0.6 \mathrm{~s}$, a load step of $750 \mathrm{~W}$ is commanded. For this load value. the emulator trips for the P method, while it continues its operation for the rest of the methods. At $t=1 \mathrm{~s}$, when a $1 \mathrm{~kW}$ load is applied, the emulator trips for the P+Obs and PDF methods, being still responsive when $\mathrm{PDF}+\mathrm{Obs}$ is used for the compensation. This demonstrates the extended range of operation allowed by the proposed observer-based methods for transient frequency drift compensation. 

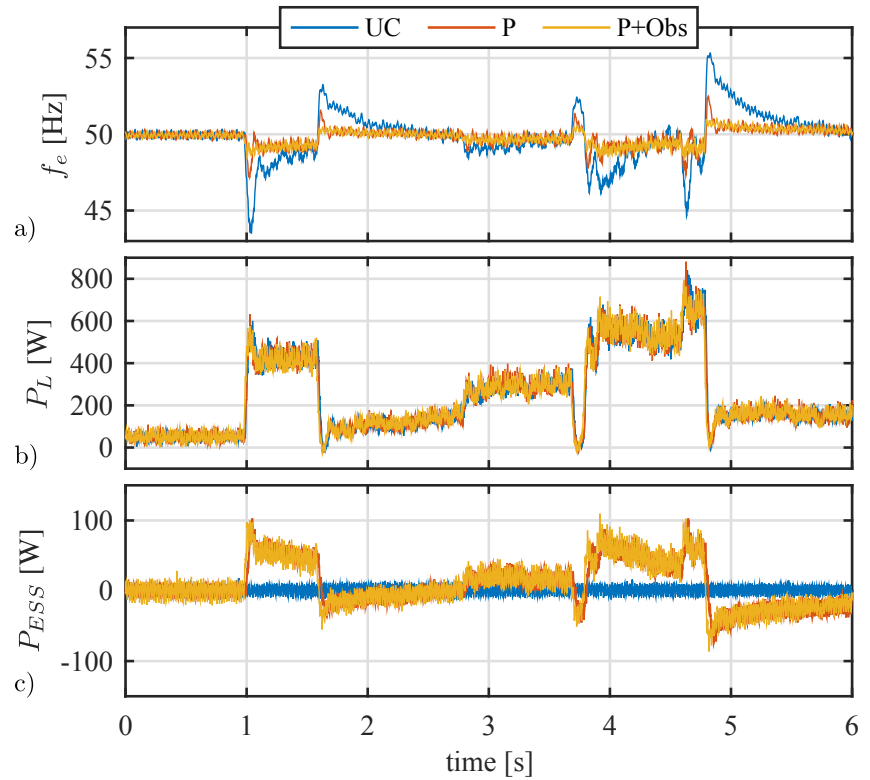

Fig. 19. Experimental results using the dynamic load emulator. a) grid frequency obtained with the drive resolver. b) load power consumption. c) active power injected by the compensator. $P$ regulator and $P+O b s$ are compared.
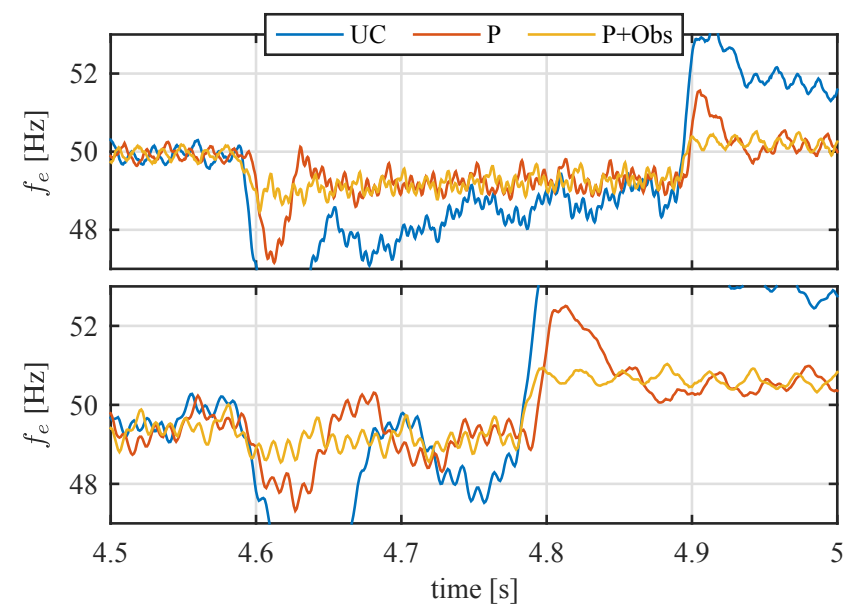

Fig. 20. Detailed view of the experiment in Fig.19. Top: time zoom from $t=0.5 \mathrm{~s}$ to $t=1.5 \mathrm{~s}$. Bottom: time zoom from $t=4.5 \mathrm{~s}$ to $t=5 \mathrm{~s}$.

\section{CONCLUSION}

In this paper, a transient frequency drift compensation has been proposed based on the use of a nearly-zero lag transient frequency estimation using a Luenberger observer. The proposed solution addresses two of the main limitations of existing frequency compensation methods in the literature: the phase lag on the frequency estimation, and the transient detection for decoupling the grid reference frequency. The performance achieved by feedback control has been improved by the development of a transient observer, formed by a novel transient detection method, based on signal correlation that effectively decouples the grid reference frequency from the compensator inputs, and a Luenberger-based observer that provides a fast frequency estimation and allows to increase the phase margin in the frequency controller. Moreover, the de-
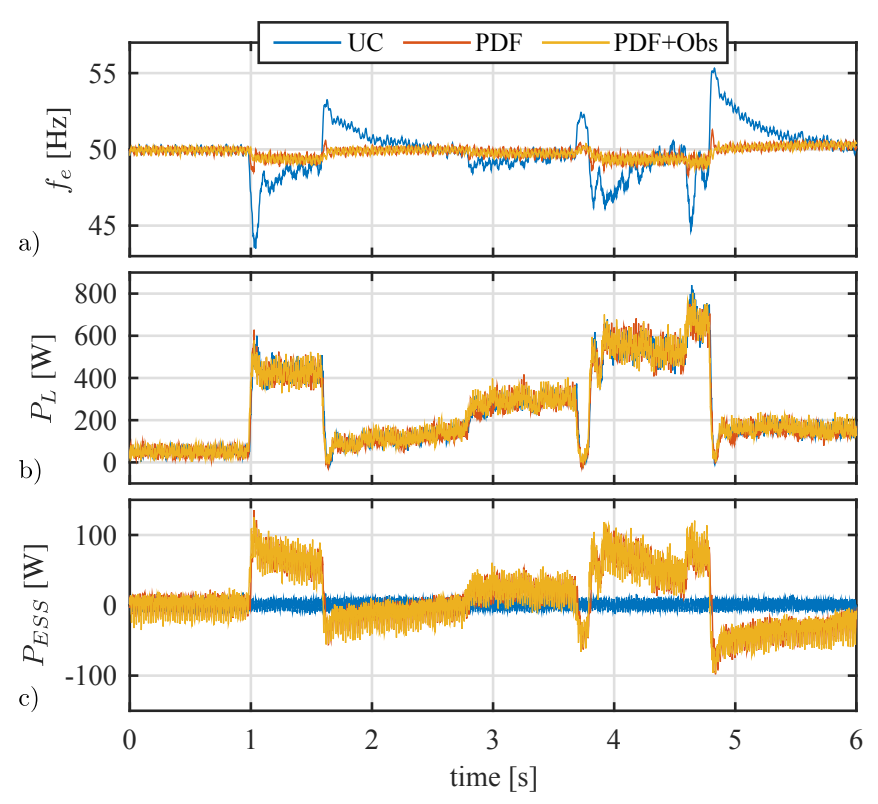

Fig. 21. Experimental results using the dynamic load emulator. a) grid frequency obtained with the drive resolver. b) load power consumption. c) active power injected by the compensator. $P D F$ regulator and $P D F+O b s$ are compared.

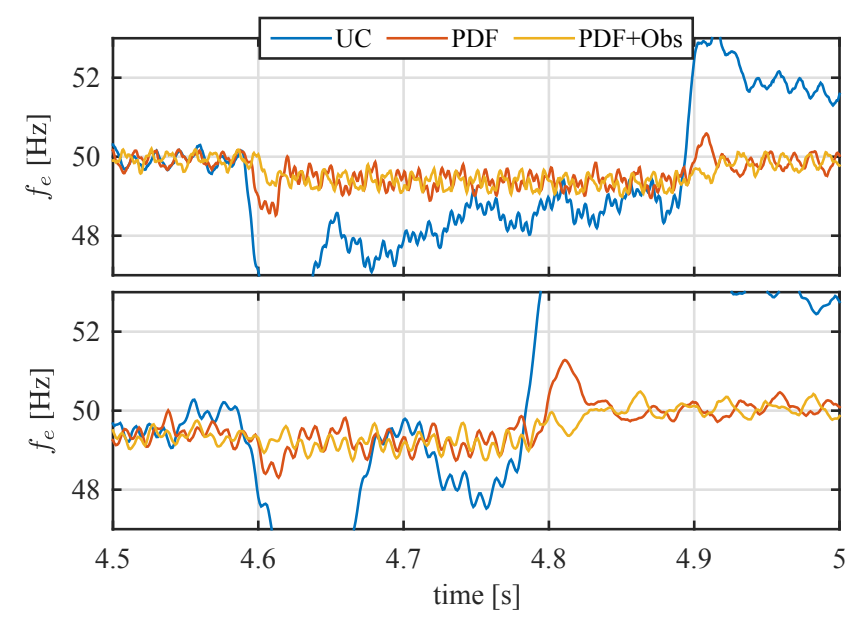

Fig. 22. Detailed view of the experiment in Fig.21. Top: time zoom from $t=0.5 \mathrm{~s}$ to $t=1.5 \mathrm{~s}$. Bottom: time zoom from $t=4.5 \mathrm{~s}$ to $t=5 \mathrm{~s}$.

veloped observer structure allows to estimate the load torque, that can be employed as a feed-forward mechanism for the decoupling of the load disturbance. A detailed comparison, including analytical derivations, simulation and experimental results, have been carried out. The methods have been tested under two different scenarios considering passive and active loads based on resistors and PEC respectively. The analytical derivations, the simulation and the experimental results have demonstrated the effectiveness of the proposed observerbased TFDC. Additionally, it has been demonstrated that the proposed method allows for an extended range operation, withstanding higher disturbances and improving the system stability. The decoupling of the grid frequency reference makes the method suitable for the operation in weak grids under droop control. Finally, a comparative evaluation of 


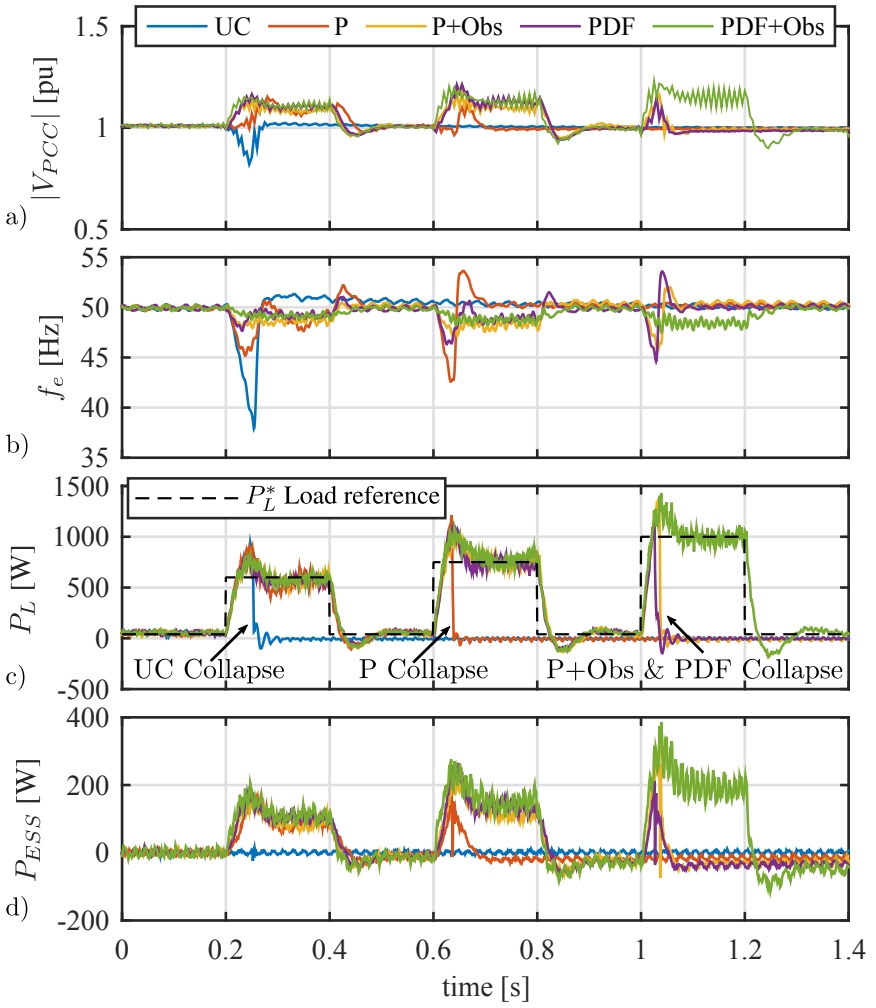

Fig. 23. Evaluation of the compensation methods under different load step disturbances introduced by the dynamic load emulator. a) Voltage magnitude at the PCC. b) Grid frequency obtained with the drive resolver. c) Load power consumption. d) Active power injected by the compensator.

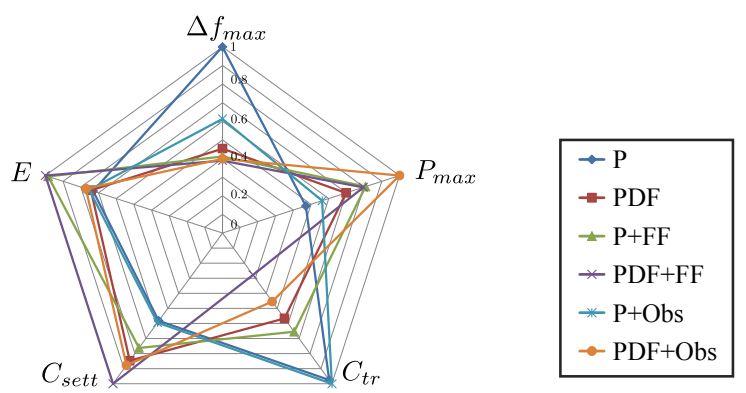

Fig. 24. Merit figure. Performance evaluation for all the considered methods. $\Delta f_{\max }:$ maximum frequency deviation, $P_{\max }$ : peak power $E$ : total shared energy, $C_{t r}$ : initial transient deviation, $C_{\text {sett }}$ : settling time for transient frequency drift.

the analyzed techniques has been done, summarizing their performance in Fig. 24. Five indexes are considered. The indexes have been derived from the data obtained in the first scenario using a resistive load and are normalized in p.u., being all of them the higher the worst.

\section{REFERENCES}

[1] J. M. Guerrero, "Editorial Special Issue on Power Electronics for Microgrids-Part II," IEEE Transactions on Power Electronics, vol. 26, no. 3, pp. 659-663, March 2011.

[2] J. Guerrero, P. C. Loh, T.-L. Lee, and M. Chandorkar, "Advanced Control Architectures for Intelligent Microgrids-Part II: Power Quality, Energy Storage, and AC/DC Microgrids," IEEE Transactions on Industrial Electronics, vol. 60, no. 4, pp. 1263-1270, April 2013.
[3] A. Agbedahunsi, M. Sumner, E. Christopher, A. Watson, A. Costabeber, and R. Parashar, "Frequency control improvement within a microgrid, using enhanced STATCOM with energy storage," in 6th IET International Conference on Power Electronics, Machines and Drives (PEMD 2012), March 2012, pp. 1-6.

[4] A. Arulampalam, J. Ekanayake, and N. Jenkins, "Application study of a STATCOM with energy storage," IEEE Proceedings on Generation, Transmission and Distribution, vol. 150, no. 3, pp. 373-384, May 2003.

[5] M. Falvo, L. Martirano, and D. Sbordone, "D-STATCOM with energy storage system for application in Smart Micro-Grids," in 2013 International Conference on Clean Electrical Power (ICCEP), June 2013, pp. 571-576.

[6] M. Beza and M. Bongiorno, "Comparison of two control approaches for stability enhancement using STATCOM with active power injection capability," in Energy Conversion Congress and Exposition (ECCE), 2015 IEEE, Sept 2015, pp. 4721-4728.

[7] M. Holmberg, M. Lahtinen, J. McDowall, and T. Larsson, "SVC Light with energy storage for frequency regulation," in 2010 IEEE Conference on Innovative Technologies for an Efficient and Reliable Electricity Supply (CITRES), Sept 2010, pp. 317-324.

[8] I. Serban and C. Marinescu, "Control Strategy of Three-Phase Battery Energy Storage Systems for Frequency Support in Microgrids and with Uninterrupted Supply of Local Loads," Power Electronics, IEEE Transactions on, vol. 29, no. 9, pp. 5010-5020, Sept 2014.

[9] H.-P. Beck and R. Hesse, "Virtual synchronous machine," in Electrical Power Quality and Utilisation, 2007. EPQU 2007. 9th International Conference on, Oct 2007, pp. 1-6.

[10] Q.-C. Zhong and G. Weiss, "Synchronverters: Inverters That Mimic Synchronous Generators," Industrial Electronics, IEEE Transactions on, vol. 58, no. 4, pp. 1259-1267, April 2011.

[11] M. Torres and L. Lopes, "Frequency control improvement in an autonomous power system: An application of virtual synchronous machines," in Power Electronics and ECCE Asia (ICPE ECCE), 2011 IEEE 8th International Conference on, May 2011, pp. 2188-2195.

[12] Y. Hirase, O. Noro, K. Sugimoto, K. Sakimoto, Y. Shindo, and T. Ise, "Effects of suppressing frequency fluctuations by parallel operation of virtual synchronous generator in microgrids," in Energy Conversion Congress and Exposition (ECCE), 2015 IEEE, Sept 2015, pp. 36943701.

[13] A. Navarro-Rodriguez, P. Garcia, R. Georgious, and J. Garcia, "A communication-less solution for transient frequency drift compensation on weak microgrids using a d-statcom with an energy storage system," in 2015 IEEE Energy Conversion Congress and Exposition (ECCE), Sept 2015, pp. 6904-6911.

[14] M. Ayar, S. Obuz, R. Trevizan, A. Bretas, and H. Latchman, "A distributed control approach for enhancing smart grid transient stability and resilience," IEEE Transactions on Smart Grid, vol. PP, no. 99, pp. $1-1,2017$.

[15] E. Hammad, A. Farraj, and D. Kundur, "On effective virtual inertia of storage-based distributed control for transient stability," IEEE Transactions on Smart Grid, vol. PP, no. 99, pp. 1-1, 2017.

[16] S. D'Arco and J. A. Suul, "Equivalence of virtual synchronous machines and frequency-droops for converter-based microgrids," IEEE Transactions on Smart Grid, vol. 5, no. 1, pp. 394-395, Jan 2014.

[17] S.-K. Chung, "A phase tracking system for three phase utility interface inverters," IEEE Transactions on Power Electronics, vol. 15, no. 3, pp. 431-438, May 2000.

[18] P. Roncero-Sanchez, X. del Toro Garcia, A. Torres, and V. Feliu, "Fundamental Positive- and Negative-Sequence Estimator for Grid Synchronization Under Highly Disturbed Operating Conditions," IEEE Transactions on Power Electronics, vol. 28, no. 8, pp. 3733-3746, Aug 2013.

[19] P. Rodriguez, A. Luna, M. Ciobotaru, R. Teodorescu, and F. Blaabjerg, "Advanced Grid Synchronization System for Power Converters under Unbalanced and Distorted Operating Conditions," in 32nd Annual Conference on IEEE Industrial Electronics, IECON 2006, Nov 2006, pp. $5173-5178$

[20] M. Torres L, L. Lopes, L. Moran T, and J. Espinoza C, "Self-Tuning Virtual Synchronous Machine: A Control Strategy for Energy Storage Systems to Support Dynamic Frequency Control," Energy Conversion, IEEE Transactions on, vol. 29, no. 4, pp. 833-840, Dec 2014.

[21] L.-Y. Lu and C.-C. Chu, "Consensus-Based Secondary Frequency and Voltage Droop Control of Virtual Synchronous Generators for Isolated AC Micro-Grids," Emerging and Selected Topics in Circuits and Systems, IEEE Journal on, vol. 5, no. 3, pp. 443-455, Sept 2015.

[22] J. Alipoor, Y. Miura, and T. Ise, "Distributed generation grid integration using virtual synchronous generator with adoptive virtual inertia," in 
Energy Conversion Congress and Exposition (ECCE), 2013 IEEE, Sept 2013, pp. 4546-4552.

[23] S. Pulendran and J. E. Tate, "Energy storage system control for prevention of transient under-frequency load shedding," IEEE Transactions on Smart Grid, vol. 8, no. 2, pp. 927-936, March 2017.

[24] J. Liu, Y. Miura, H. Bevrani, and T. Ise, "Enhanced virtual synchronous generator control for parallel inverters in microgrids," IEEE Transactions on Smart Grid, vol. 8, no. 5, pp. 2268-2277, Sept 2017.

[25] D. Luenberger, "Observing the State of a Linear System," IEEE Transactions on Military Electronics, vol. 8, no. 2, pp. 74-80, April 1964.

[26] "IEEE Standard for Interconnecting Distributed Resources with Electric Power Systems - Amendment 1," IEEE Std 1547a-2014 (Amendment to IEEE Std 1547-2003), pp. 1-16, May 2014.

[27] S.-K. Sul, Control of Electric Machine Drive Systems. Wiley IEEE press, 2011.

[28] M. Torres L, "Dynamic frequency control in diesel-hybrid autonomous power systems using virtual synchronous machines," Ph.D. dissertation, Concordia University, Montreal, Quebec, Canada, May 2013.

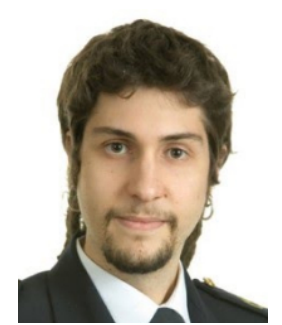

Ángel Navarro-Rodríguez (S'15) received the B.Sc. degree in telecommunications engineering with honors from the University of Castilla LaMancha, Spain in 2012 and the M.Sc. degree in Electrical Energy Conversion and Power Systems from the University of Oviedo, Gijon, Spain in 2014. Nowadays he is Ph.D student in the Department of Electrical, Computer, and Systems Engineering in the University of Oviedo, granted by the government of Principado de Asturias. He is part of the LEMUR research team in University of Oviedo since 2014 and his research interests include Energy Storage Systems, Control Systems, Electronics, Power Electronic Converters, Microgrids, power quality and Renewable Energies.

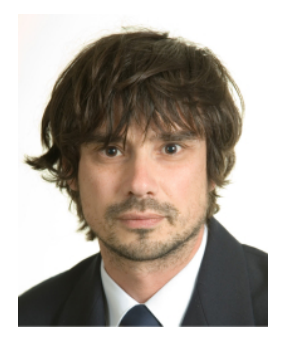

Pablo García (S01-A06-M09) received the M.S. and $\mathrm{Ph} . \mathrm{D}$. degrees in electrical engineering and control from the University of Oviedo, Gijón, Spain, in 2001 and 2006, respectively. In 2004, he was a Visitor Scholar at the University of Madison-Wisconsin, Madison, WI, USA, Electric Machines and Power Electronics Consortium. In 2013 he was a visiting scholar at The University of Nottingham, UK. He is the co-author of more than 20 IEEE journals and 50 international conferences. He is currently an Associate Professor within the Department of Electrical, Electronics, Computer, and Systems Engineering at the University of Oviedo. His research interests include control of grid-tied converters, parameter estimation, energy conversion, AC drives, sensorless control, AC machines diagnostics and signal processing.

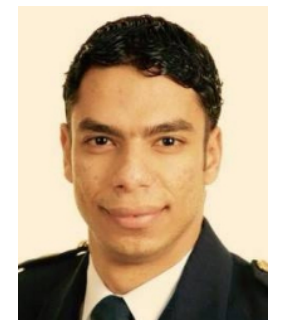

Ramy Georgious (S'15) received the B.Sc. degree from Port Said University, Port Said, Egypt, in May 2010, in Electrical Engineering with average appreciation Excellent with honors degree. He received the M.Sc. degree from University of Oviedo, Gijon, Asturias, Spain, in June 2014, in Electrical Energy Conversion and Power Systems (EECPS). He worked as a teacher assistant in the Electrical Engineering department in Port Said University from March 2011 to September 2012. He joined LEMUR research team in University of Oviedo in July 2014. He was granted by the government of Principado de Asturias to do his $\mathrm{Ph} . \mathrm{D}$. in the University of Oviedo. His research interests include Energy Storage Systems, Control Systems, Power Electronic Converters, Microgrids, Renewable Energy, Design and Simulation.

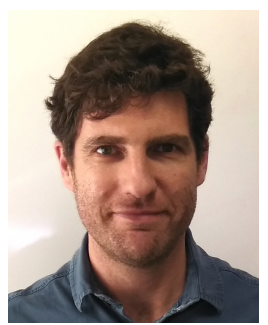

Jorge García (S'01-M'05-SM'11), received the M.Sc. and $\mathrm{PhD}$. degrees in electrical engineering from the University of Oviedo, Asturias, Spain, in 2000 and 2003, respectively. In December 1999, he joined the Electrical and Electronic Engineering Department of the University of Oviedo, where he is currently an Associate Professor. He works with the LEMUR research team; his current research interests include power electronics and control for industrial, grid support and lighting applications. Dr. Garcia is co-author of more than 40 journal papers and more than 80 international conference papers in power and industrial electronics. Dr. Garcia was a recipient of the University of Oviedo Outstanding PhD. Thesis Award in 2005.

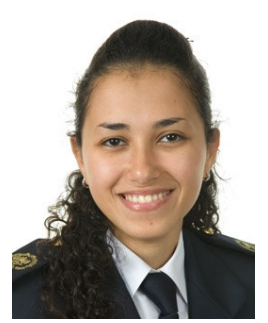

Sarah Saeed (S'16) Received B.Sc. degree in Electrical Engineering from the University of Ain Shams, Egypt, in 2013. She received her M.Sc. degree in Electrical Energy Conversion and Power Systems in 2015. She received the first prize for her master thesis awarded by the chair of Mobility in the University of Oviedo in 2016. She is currently working towards her $\mathrm{PhD}$ degree from the University of Oviedo as an investigator within LEMUR research group, and granted by the government of the Principado de Asturias. Her research interests include: power electronic converters modeling and digital control, design and construction of DC/DC converter topologies, integration of energy storage systems in power electronic applications, and nonlinear magnetics. 